TM-1531

[SSC-N-526]

\title{
Conceptual Design of a Superconducting Solenoid for a Magnetic SSC Detector*
}

\author{
R. W. Fast, J. H. Grimson, R. D. Kephart, H. J. Krebs, M. E. Stone, \\ D. Theriot, and R. H. Wands \\ Fermi National Accelerator Laboratory \\ P.O. Box 500, Batavia, Mlinois 60510
}

July 1988

*Paper delivered at the 12th International Cryogenic Engineering Conference, Southampton, England, July $12-15,1988$, and submitted for publication in the conference proceedings. 


\title{
CONCEPTUAL DESIGN OF A SUPERCONDUCTING SOLENOID FOR A MAGNETIC SSC DETECTOR
}

R.W. Fast, J.H. Grimson, R.D. Kephart, H.J. Krebs, M.E. Stone, D. Theriot, and R.H. Wands

Fermi Nátional Accelerator Laboratory, Batavia, nlinois, USA

\begin{abstract}
The conceptual design of a large superconducting solenoid suitable for a magnetic detector at the Superconducting Super Collider (SSC) has begun at Fermilab. The magnet will provide a magnetic field of $2 \mathrm{~T}$ over a volume $8 \mathrm{~m}$ in diameter by $16 \mathrm{~m}$ long. The particle-physics calorimetry will be inside the field volume and so the coil will be bath cooled and cryostable; the vessels will be stainless steel. Predictibility of performance and the ability to safely negotiate all probable failure modes, including a quench, are important items of the design philosophy. Although the magnet is considerably larger than existing solenoids of this type and although many issues of manufacturability, transportability and cost have not been completely addressed, our conceptual design has convinced us that this magnet is a reasonable extrapolation of present technology.
\end{abstract}

\section{INTRODUCTION}

A large solenoid is being considered as part of a detector (Fig. 1) for experiments performed on the Superconducting Super Collider (SSC). The conceptual design of a solenoid suitable for a detector has begun at Fermilab and some preliminary specifications have been developed. The field volume proposed to perform proton-proton experiments with the SSC is $8 \mathrm{~m}$ in diameter and $16 \mathrm{~m}$ long; a field of 1.5 to $2 \mathrm{~T}$ is desired. The magnet will have an iron flux return yoke at a field of $1.5 T$. $A$ significant feature of this detector is that all the calorimetry will be inside the bore of the magnet. This feature impacts the design of both the calorimeter support system and the superconducting coil in a major way. Another feature of the detector that influences the design of the magnet is its location in a detector hall 100 to $150 \mathrm{~m}$ below the surface.

The calorimeter and central tracking chamber for the SSC detector will occupy the entire field volume and will weigh about 5000 tonnes. This weight could be transmitted to the floor of the detector hall either through the vacuum vessel of the magnet coil or through an independent support structure. The calorimeter will be split at the longitudinal center for instrumentation cabling. Taking advantage of this, we have designed two independent calorimeter support structures, each $8 \mathrm{~m}$ long, supported to the iron yoke at both ends. The stainless steel weldment consists of two cylinders with a web of trusses between; it has a radial thickness of $250 \mathrm{~mm}$. The calorimeter modules will rest on rails along each side of the structure and can be installed from either or both ends.

Because the calorimetry is inside the bore of the solenoid it is not necessary that the coil and ressels be thin in terms of radiation and absorption lengths. We therefore chose a cryostable, bath-cooled coil, able to withstand quenching without damage. The conductor is copper stabilized; the helium and vacuum vessels are stainless steel. We have located as many of the active elements as possible in an aboveground service building; the power supply; dump switches, pumps and control valves. 
For the purpose of this conceptual study, we have adopted a fabrication and assembly scenario as follows: (1) The coil is wound in 2-m long modules about a vertical axis, each module forming a liquid helium vessel. (2) Four coil modules are stacked vertically and bolted together to form an $8-\mathrm{m}$ cold mass; electrical interconnections are made in liquid helium pipes along the top. (3) The outer vacuum shell, with a liquid nitrogen cooled thermal shield attached, is lowered over the cold mass and the supports attached to each end. (4) The inner vacuum shell-thermal shield subassembly is vertically inserted and the flat annular heads welded in place. (5) The assembly is then rotated to a horizontal axis and a 5000-L helium storage dewar attached. (6) The two 8-m"assemblies are cryogenically and electrically tested. (7) The 500-tonne assemblies are then lowered into the detector hall and secured to the iron yoke in a way that allows for a $20^{\circ} \mathrm{C}$ thermal contraction of the $8-\mathrm{m}$ vacuum vessel. Figure 2 is a cross section of the coil and cryostat.

\section{CONDUCTOR AND QUENCH PROTECTION}

The inner diameter of the superconducting coil was chosen as $9.5 \mathrm{~m}$, which provides adequate space for vessel shells, insulating vacuum and clearance to the calorimeter support structure. We chose to operate at a current of $5 \mathbf{k A}$ and to electrically connect four 2-m coil modules in series with superconducting bus. Table 1 gives some. of the operating parameters of the magnet. We believe that it is essential that the coil survive quenches without damage. We will provide this protection through the use of an external fast dump resistor and by specifying a rather low conductor current density. The parameters associated with a quench are given in Table 2. Because of the large heat load due to eddy current in the helium vessel, a fast dump will be initiated only when a quench is detected. A normal discharge will be through a slow dump resistor, with the power supply reversed so the discharge is at constant voltage.

We selected a built-up conductor with a $\mathrm{Nb}-\mathrm{Ti} / \mathrm{Cu}$ monolith or cable soldered into additional copper stabilizer. The conductor dimensions are $16 \mathrm{~mm} \times 18 \mathrm{~mm}$. With a short sample rating of $10 \mathrm{kA}_{2}$ at $3 \mathrm{~T}$ and $4.5 \mathrm{~K}$ and a current density in the superconductor of $3 \times 10^{5} \mathrm{~A} / \mathrm{cm}^{2}$, the copper to superconductor ratio will be about 90 . The surface heat flux with a copper RRR of 100 and $25 \%$ surface wetting is 0.05 $\mathrm{W} / \mathrm{cm}^{2}$.

\section{COIL-HELIUM VESSEL MODULE}

Each 2-m coil module has seven layers of 93 turns for a total of 651 turns. We chose G-10 buttons on a string as the turn-to-turn insulation, slotted G-10 sheets between layers and slotted and channeled G-10 and Kapton as insulation to ground, giving a packing factor of 0.65 . The conductor is layer wound the "easy" way, beginning at the outer layer, on the inside of a coil form which makes up the outer shell and flat heads of the helium vessel. The winding fixture applies the radial preload; compression bars at one end of the coil apply the axial preload. The coil module will be closed by welding the inner shell to the coil form.

The 2-m coil-helium vessel module is designed for a gauge pressure of $0.7 \mathrm{MPa}$ (100 psi). The inner and outer shells are also designed for the axial electromagnetic force. The radial electromagnetic pressure of $1.6 \mathrm{MPa}(230 \mathrm{psi})$ is partially resisted by the conductor and partially by the outer shell, depending on the stiffness of the coil. The vessel is adequately relieved for a loss of insulating vacuum or a quench.

We propose a thermosiphon to provide a flow of liquid helium through the coil package. Each of the coil-helium vessel modules has a supply line from the storage dewar leading to the bottom of the module. These lines are very well insulated so they contain only liquid helium. Two return and vent lines lead from the top of each module to the storage dewar. 
When installed in the iron yoke, each 8 -m cold mass has an axial body force on it which depends on the geometry of the end wall and reentrant end plug. For the one coil configuration studied thus far, calculations with a cylindrically symmetric iron flux return indicate that this force is a minimum without reentrant iron, being about 7.3 MN (1.64 $\left.\times 10^{6} \mathrm{lb}\right)$ toward the symmetry plane. The axial force constant is 149 $\mathrm{MN} / \mathrm{m}\left(8.5 \times 10^{5} \mathrm{lb} / \mathrm{in}\right)$ and the radial force constant is $8.8 \mathrm{MN} / \mathrm{m}\left(5 \times 10^{4} \mathrm{lb} / \mathrm{in}\right)$; from experience with the solenoid at the Collider Detector-Fermilab we have chosen an axial and radial misalignment or the equivalent, due to non-uniform iron properties, of $25 \mathrm{~mm}$. The support system is not designed for independent charging or operation of the two 8-m coil modules; bumpers limit axial travel if only one module is energized because of a control system failure.

The system to support the 8-m cold mass in the vacuum vessel could have either separate elements to react the axial and radial force components or combined-function elements. We have tentatively chosen the latter. We believe that the supports should be metallic, possibly Inconel 718, with spherical bearings on each end. The attachment points on the vacuum vessel are near the ends to avoid carrying the electromagnetic forces through the vacuum shells. Each support will have an intercept cooled by a forced flow of subcooled liquid nitrogen at about $80 \mathrm{~K}$ and a liquid helium intercept in a thermosiphon circuit independent of the coil module circuit.

\section{REFRIGERATION SYSTEM}

The refrigeration plant is located at ground level, with vacuum jacketed lines going down to the detector hall. The helium plant supplies liquid to the storage dewars on top of the detector. Because of the depth of the detector hall a cold compressor is used to maintain the liquid in the dewars at about $30 \mathrm{kPa}$ (4.5 psig) and $4.5 \mathrm{~K}$. For the proposed electrical insulation scheme and resulting flow paths through the coil, the helium supply and return lines can be sized to provide a thermosiphon flow rate through each $2-\mathrm{m}$ coil module of at least $23 \mathrm{~g} / \mathrm{s}$ with a return gas fraction less than $1 \%$ by weight. The flow rate through the support intercept circuit will be about 50 $\mathrm{g} / \mathrm{s}$ at a gas fraction of $7 \%$ or less. The total expected heat load to the helium system is $360 \mathrm{~W}$ plus $36 \mathrm{~L} / \mathrm{h}$ (540 W equivalent). The 4.5-K helium refrigerator will probably have a capacity of 1600 to $1800 \mathrm{~W}$. Sub-cooled liquid nitrogen at an average temperature of $83 \mathrm{~K}$ is forced through the various shield and intercept circuits by a circulator pump at detector hall level. The total heat load to the nitrogen system is expected to be $6 \mathrm{~kW}$.

The total cold mass (two 8-m assemblies) is about 800 tonnes; an overall cooldown time of about two weeks is desired. A separate cooldown refrigerator, consisting of a helium to liquid nitrogen heat exchanger and a turboexpander, providing $400 \mathrm{~g} / \mathrm{s}$ of $55 \mathrm{~K}$ helium gas can achieve this.

\section{CONCLUSIONS}

It appears that the diameter of this detector solenoid will preclude its fabrication at a vendor's off-site facility, although some components and subassemblies, e.g. the coil form, could possibly be fabricated elsewhere and shipped by barge and helicopter. $A$ large hall will be necessary on the SSC site for the coil winding, the closure welding of the coil-helium vessel modules, the assembly of the $8-\mathrm{m}$ modules and the testing with the refrigerator. The hall will require at least a 100 tonne crane with a hook height of at least $12 \mathrm{~m}$. The access shaft from the surface down 100-150 $\mathrm{m}$ to the detector hall will have to be about $14 \mathrm{~m}$ in diameter.

We concluded, as a result of this preliminary study, that the magnet is a reasonable extrapolation of superconducting magnet technology. Additional work on techniques for its manufacture including cost and time estimates are still needed. The optimization of 
parameters through more detailed study might result in a more cost-effective design, however at this point in the effort we see nothing that would preclude the construction of such a magnet.

\section{ACKNOWLEDGEMENTS}

This work was sponsored by Universities Research Association, Inc. under contract with the U.S. Department of Energy. The authors would like to thank R.W. Baldi and R.A. Johnson of General Dynamics/Space Systems Division for providing us with engineering reports on the MFTF solenoids which were very useful to us in developing the concepts for this magnet.

Table 1. Operating Parameters

Center-line axial field $=2 \mathrm{~T}$

Operating current $=5 \mathrm{kA}$

Total stored energy at $2 \mathrm{~T}=1 \mathrm{GJ}$

Charge time $=100 \mathrm{~min}$ with $50 \mathrm{~V}$

Slow dump resistor $=0.010$

Operating temperature $=4.5 \mathrm{~K}$
Table 2. Quench Parameters

Self-inductance $=60 \mathrm{H}$

Fast dump voltage $=500 \mathrm{~V}$

Fast dump resistor $=0.10$

Maximum hot spot temperature $=100 \mathrm{~K}$

Stabilizer current density $=1756 \mathrm{~A} / \mathrm{cm}^{2}$

Fast dump eddy current heat load $=650 \mathrm{~W}$

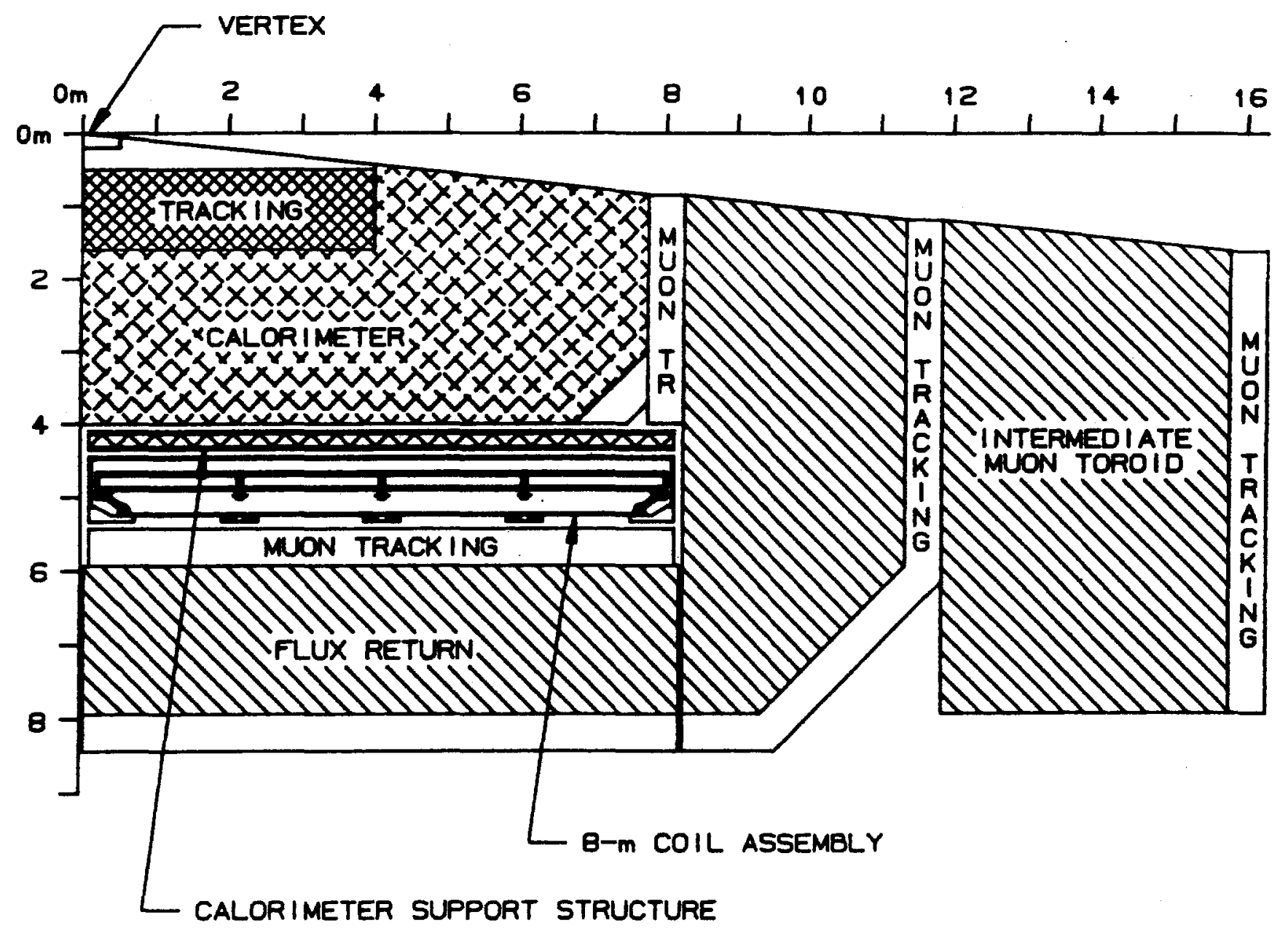

Fig. 1 SSC detector with large superconducting solenoid. 


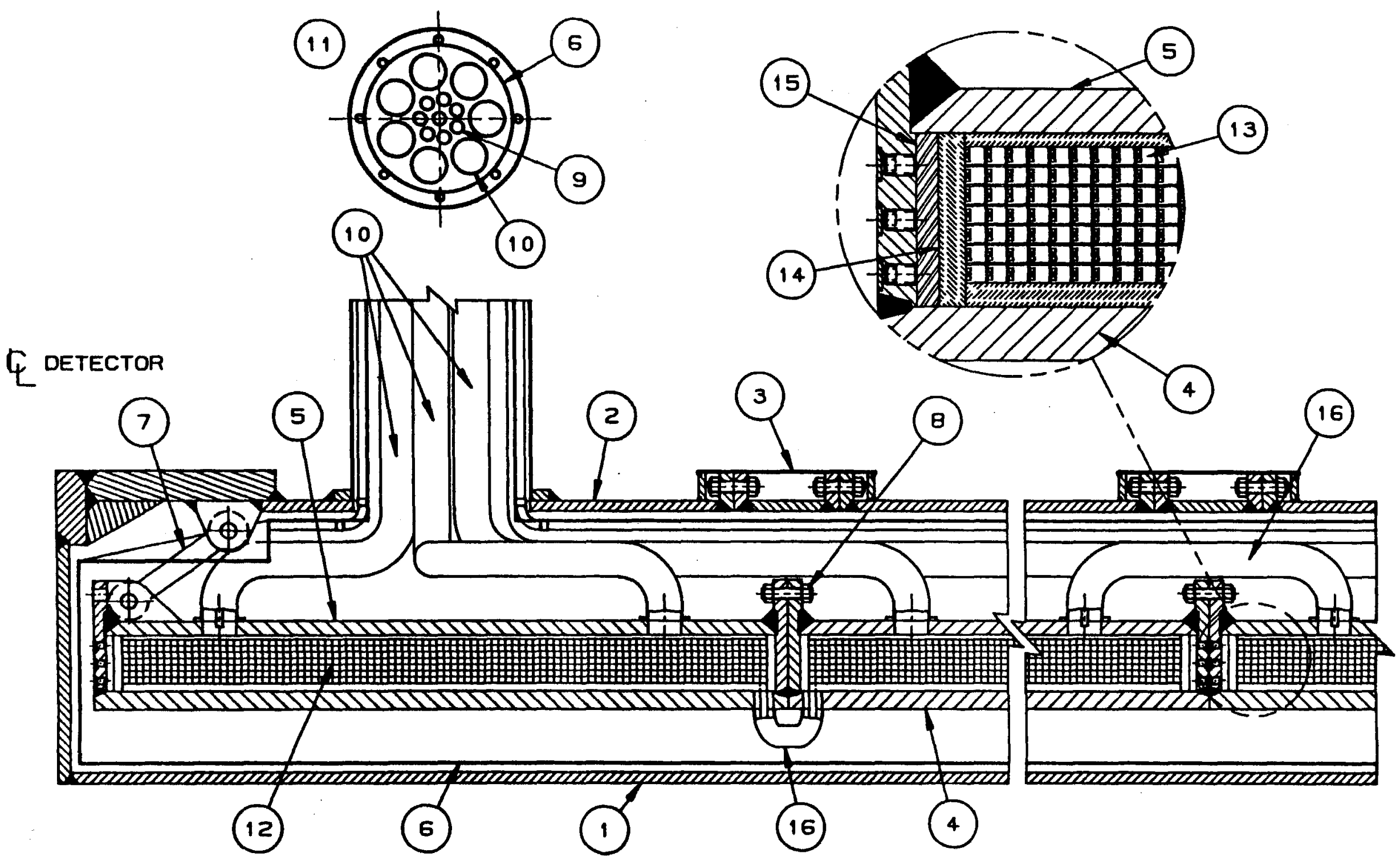

Fig. 2 Axial cross section of coil, helium and vacuum vessels: 1 , inner and 2, outer vacuum shell; 3 , assembly joint (if required); 4 , inner and 5 , outer helium vessel shell; 6 , radiation shield; 7 , cold mass support; 8 , coil module attachment; 9 , liquid helium supply pipe; 10, helium return/vent pipe; 11, chimney to storage dewar; 12, coil winding; 13, conductor; 14, G10 insulation; 15, axial preload bar; 16 , electrical interconnect pipe. 